Revista Brasil. Bot., V.33, n.2, p.343-354, abr.-jun. 2010

\title{
Própolis marrom da vertente atlântica do Estado do Rio de Janeiro, Brasil: uma avaliação palinológica
}

\author{
ALEX DA SILVA DE FREITAS ${ }^{1,4}$, ORTRUD MONIKA BARTH ${ }^{1,2} \mathrm{e}$ \\ CYNTHIA FERNANDES PINTO DA LUZ ${ }^{3}$
}

(recebido: 11 de dezembro de 2008; aceito: 29 de abril de 2010)

\begin{abstract}
Brownish propolis from the Atlantic coastal areas in the state of Rio de Janeiro, Brazil: a palynological approach). Several ecosystems appear along the coastline in Rio de Janeiro State where apicultural activities are developed for propolis production, although only a few papers have been published on palynological analysis of the propolis produced in this region. Twenty four samples of propolis were collected and analysed throughout 1997, originated from three apiaries located in distinct areas on the west Atlantic coast in the municipality of Rio de Janeiro. The palynological analysis was carried out after removal of wax and resin using ethanol, acetolysis, and counting 500 pollen grains per sample. All samples showed a predominance of Eucalyptus pollen type together with Mimosa caesalpiniaefolia apart from minor quantities of Mimosa scabrella. Cecropia was present in most samples but the percentages varied considerably. Anacardiaceae (four pollen types), Asteraceae, Citrus, Cocos and Poaceae also occurred in most samples, but in lower quantities. The original vegetation in this region (Atlantic forest and "restinga") was represented by a few pollen types with percentages below 3\% (Astronium, Casearia, Celtis, Mansoa/Sparattosperma, Myrcia, Schinus and Tabebuia). Statistical analysis reflected the correlation between the plant species identified by their pollen grains and the studied areas. The palynological analysis of the brownish propolis showed mainly the similarity of the pollen spectra in these three areas, making evident the altered vegetation (from degraded areas and cultivation).
\end{abstract}

Key words - Apis mellifera, palynological analysis, propolis, Rio de Janeiro, vegetation

RESUMO - (Própolis marrom da vertente atlântica do Estado do Rio de Janeiro, Brasil: uma avaliação palinológica). Diversos ecossistemas são encontrados ao longo da área litorânea do Estado do Rio de Janeiro onde se desenvolvem atividades apícolas visando à produção de própolis, entretanto, poucos são os trabalhos que tratam da análise palinológica da própolis dessa região. Foram analisadas vinte e quatro amostras de própolis coletadas ao longo do ano de 1997 e procedentes de três apiários localizados em áreas distintas da vertente atlântica na zona oeste do município do Rio de Janeiro. As análises palinológicas foram realizadas a partir da remoção da cera e resina com etanol e, pelo uso da acetólise, contando-se 500 grãos de pólen por amostra. Em todas as amostras houve a predominância do tipo polínico Eucalyptus em conjunto com Mimosa caesalpiniaefolia, além de Mimosa scabrella que, no entanto, foi observado com valores mais baixos. Cecropia esteve presente na maioria das amostras, mas seus percentuais variaram muito. Anacardiaceae (quatro tipos polínicos), Asteraceae, Citrus, Cocos e Poaceae também ocorreram na maioria das amostras, mas sempre com baixos valores. As formações vegetais originais da região (mata atlântica e restinga) foram representadas por alguns tipos polínicos com percentuais abaixo de 3\% (Astronium, Casearia, Celtis, Mansoa/Sparattosperma, Myrcia, Schinus e Tabebuia). As análises estatísticas refletiram a correlação entre as espécies de plantas reconhecidas através de seus grãos de pólen e as áreas de estudo. A análise palinológica da própolis marrom demonstrou principalmente a semelhança dos espectros polínicos nessas três áreas, evidenciando a vegetação alterada (de áreas degradadas e cultivo).

Palavras-chave - análise palinológica, Apis mellifera, própolis, Rio de Janeiro, vegetação

\section{Introdução}

As abelhas Apis mellifera L. preparam a própolis a partir de uma mistura de exsudatos resinosos recolhidos

1. Universidade Federal do Rio de Janeiro, Instituto de Biologia, Departamento de Botânica, Laboratório de Palinologia, 21949-900 Rio de Janeiro, RJ, Brasil.

2. Instituto Oswaldo Cruz, Avenida Brasil 4365, 21040-900 Rio de Janeiro, RJ, Brasil.

3. Secretaria do Meio Ambiente de São Paulo, Instituto de Botânica, Núcleo de Pesquisa em Palinologia, Avenida Miguel Stéfano 3687, Água Funda, 04301-902 São Paulo, SP, Brasil.

4. Autor para correspondência: alex_silvafreitas@yahoo.com.br de várias estruturas vegetais, cera de abelhas, óleos essenciais extraídos de flores e detritos de madeira, sendo cerca de $5 \%$ de seu peso formado por pólen que participa como contaminante no produto (Ghisalberti 1979, Warakomska \& Maciejewicz 1992, Barth et al. 1999). É produzida em qualquer época do ano sendo utilizada pelas abelhas com o intuito mecânico de vedar e calafetar a colméia, atuando como um agente termorregulador, evitando a exposição da colônia a correntes de ar e infestação por elementos estranhos. Por apresentar propriedades antissépticas, serve na assepsia da colônia, pois impede a ação de microrganismos, principalmente nas áreas de postura de cria e para embalsamar insetos 
invasores (Marcucci 1995, Kujumgiev et al. 1999, Banskota et al. 2001, Manrique \& Soares 2002, Teixeira et al. 2003).

No Brasil existe uma grande amplitude de tipos de própolis constituindo-se o país, atualmente, no terceiro produtor mundial (Teixeira et al. 2003). Algumas própolis são fibrosas e firmes, enquanto outras são gomosas e maleáveis, possuindo composição química complexa. Sua coloração pode variar do amarelo claro, marrom esverdeado ao negro, dependendo da vegetação de origem e dos fatores ambientais do local de produção (Marcucci et al. 2001, Salatino et al. 2005).

Considerando-se a diversidade da flora brasileira potencialmente produtora de matéria-prima para produção de própolis, fica evidente que os conhecimentos científicos acerca da sua origem botânica e geográfica precisam ser expandidos (Teixeira et al. 2003, Barth 2004). Com a crescente variedade e disponibilidade dos derivados apícolas no mercado brasileiro e o interesse internacional, incentiva-se a pesquisa de caracterizar palinologicamente os diversos tipos de própolis para que o controle da qualidade deste produto tenha uma melhor resolução, ampliando-se as perspectivas comerciais no Brasil e para o exterior (Luz et al. 2007a).

O espectro polínico resultante do resíduo insolúvel da própolis é composto em sua maioria pelo pólen trazido pelas abelhas e pelo pólen anemófilo que aderiu à resina. Desta maneira, a análise palinológica é um instrumento valioso na determinação de sua origem geográfica, o que permite fazer a distinção entre as diferentes regiões produtoras, podendo elucidar também sobre a estação do ano em que foi elaborada, oferecendo resultados que podem ser usados para a verificação e rotulagem das amostras (D’Albore 1979, Ghisalberti 1979, Barth 1998, Barth et al. 1999). A caracterização de outros elementos orgânicos e inorgânicos (elementos figurados), em conjunto com o pólen, fornece dados complementares para a distinção entre amostras. Foi observada em amostras de própolis a presença de epiderme com seus anexos como estômatos, pêlos vegetais (tricomas) e glândulas, por alguns autores como Warakomska \& Maciejewicz (1992), Barth (1998), Bastos (2001) e Montenegro et al. (2000, 2001). Distingue-se de produto semelhante elaborado pelas abelhas nativas sem ferrão, denominado geoprópolis (Nogueira Neto 1997, Barth \& Luz 2003, Barth 2006) o qual não contém tricomas, mas sedimento de terra ou barro usado na sua elaboração (Barth 1998, Barth et al. 1999).

No Brasil, Barth (1998) e Barth et al. (1999) analisaram palinologicamente algumas amostras de própolis marrom das regiões Sudeste e Sul do Brasil, demonstrando que os tipos polínicos entomófilos Eucalyptus e Eupatorium, assim como o anemófilo Cecropia, estiveram melhor representados quantitativamente, sendo comum ocorrerem dois tipos de pêlos vegetais (tricomas tectores e glandulares), além de esporos e filamentos de fungos. Já Bastos et al. (2000) e Bastos (2001) analisaram amostras de própolis verde de Minas Gerais, destacando a espécie Baccharis dracunculifolia DC. (Asteraceae) como principal fonte fornecedora de resina, fato comprovado pela observação de fragmentos de epiderme dos ápices foliares desta espécie, resultando na coloração esverdeada da própolis.

Pretende-se, no presente estudo, caracterizar geograficamente amostras de própolis marrom coletadas em apiários situados em três áreas distintas de vegetação na vertente costeira atlântica na zona oeste do município do Rio de Janeiro, utilizando-se do conhecimento da morfologia polínica e, comparando-as por similaridade de acordo com a composição dos tipos polínicos através da aplicação de técnicas estatísticas multivariadas, contribuindo dessa maneira para a certificação deste produto e para os estudos ecológicos com abelhas Apis mellifera no Brasil.

\section{Material e métodos}

Vinte e quatro amostras de própolis foram obtidas simultaneamente em três apiários localizados na zona oeste do município do Rio de Janeiro, na Bacia de Sepetiba (figura 1), sendo oito em Guaratiba, nove em Campo Grande e sete em Sepetiba. Esta região abrange diversos tipos de domínios ecológicos em função dos componentes geográficos, geológicos, climatológicos e uso da terra, predominando na atualidade a vegetação secundária degradada, campos ruderais, pastagens e cultivos. Em certos locais dessa Bacia ainda podem ser encontrados remanescentes da vegetação de restinga, mata atlântica, campos de várzea e manguezais (Menezes \& Araújo 2004, 2005, Peixoto et al. 2004, Menezes et al. 2005, Afonso et al. 2007).

Em relação às localidades dos apiários utilizados na presente pesquisa, estas abrangem áreas de restinga e mata pluvial atlântica (Guaratiba e Sepetiba), vegetação secundária degradada com campo ruderal e pastagem (presente em todas as localidades) e aquelas onde a vegetação nativa foi totalmente removida e introduziram-se espécies exóticas devido à densa urbanização (Campo Grande).

As coletas das amostras nos apiários foram realizadas ao longo do ano de 1997, mensalmente. Em alguns meses não foi possível realizar a coleta ora devido ao tempo chuvoso, ora por dificuldades de acompanhamento pelos apicultores. A metodologia empregada na preparação palinológica das amostras foi a de Barth (1998), realizando extração pelo 


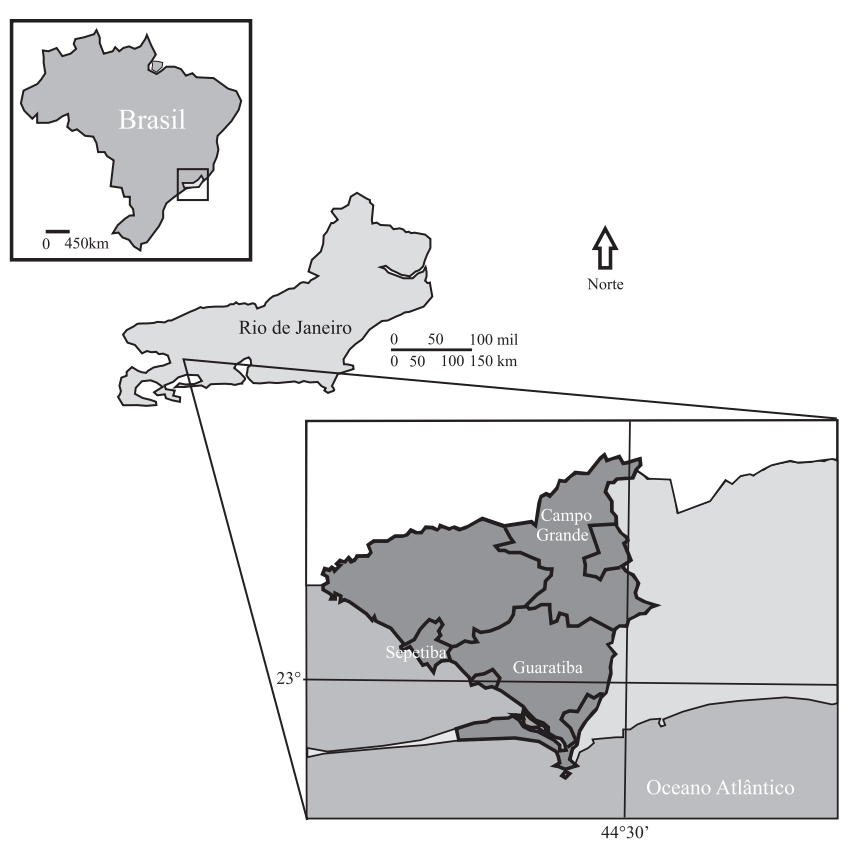

Figura 1. Mapa do Estado do Rio de Janeiro, localizando as três áreas de coleta das amostras de própolis no Município do Rio de Janeiro.

Figure 1. Map of the State of Rio de Janeiro, showing the three areas of propolis collection in the municipality of Rio de Janeiro.

etanol em 0,5 grama de própolis, seguido de fervura em $\mathrm{KOH}$, tratamento por ultrassom e filtragem por tela de 0 , $3 \mathrm{~mm}$ para eliminação de fragmentos maiores. Nesta etapa, foram montadas duas lâminas de microscopia do sedimento obtido após centrifugação para observação de pêlos vegetais e outros detritos menos resistentes ao tratamento químico consequente. Em seguida foi aplicado o método da acetólise (Erdtman 1952), preparando-se também duas lâminas de microscopia com gelatina glicerinada, uma corada com fucsina básica e outra sem corante.

Para a identificação dos tipos polínicos foi consultada a palinoteca do Laboratório de Palinologia, Departamento de Botânica (UFRJ), bem como literatura específica (Barth 1970a, b, c, d, 1989, 1998, Roubik \& Moreno 1991, Barth et al. 1999). A avaliação do percentual dos tipos polínicos baseou-se em Louveaux et al. (1978) para amostras de mel, contados 500 grãos de pólen por amostra.

A classificação dos tipos polínicos de acordo com os tipos de vegetação das áreas de estudo baseou-se nas listagens florísticas apresentadas em Peixoto et al. (2004) para o extrato arbóreo em remanescentes de mata atlântica de planície litorânea em Pedra de Guaratiba, Trevisol et al. (2002) para a vegetação espontânea de áreas degradadas da Ilha da Madeira em Itaguaí, assim como nas de Menezes \& Araújo (2004), Menezes et al. (2005) e Afonso et al. (2007) para a restinga da Marambaia.
Para verificar a similaridade entre as amostras utilizouse a análise de componentes principais (ACP) a fim de examinar a influência dos dados quantitativos e qualitativos na ordenação dos tipos polínicos. A matriz incluiu todos os tipos polínicos presentes em cada amostra com seus valores de contagem absoluta. Para a transformação da contagem absoluta pelo logaritmo natural $[\log (\mathrm{x}+1)]$ foi utilizado o programa FITOPAC (Shepherd 1996) e posteriormente realizada a ordenação através de matriz de covariância utilizando-se o programa PC-ORD 4.0 (McCune \& Mefford 1999). A variabilidade entre as amostras foi expressa utilizando-se os dois primeiros eixos da ACP. O programa MINITAB 15 (Minitab 2008) foi utilizado para a confecção do dendrograma de percentagem de similaridade entre as amostras de própolis.

\section{Resultados e discussão}

Nas amostras de própolis analisadas de Campo Grande, Guaratiba e Sepetiba foram observados no total 45 tipos polínicos pertencentes a 29 famílias e 30 gêneros (tabela 1). Os mais frequentes foram Eucalyptus (Myrtaceae) (figura 2), Mimosa caesalpiniaefolia e Mimosa scabrella (Leguminosae) (figuras 3-4). Cecropia (Urticaceae) (figura 5) esteve presente na maioria das amostras com valores variáveis. Anacardiaceae (tipo 1, Astronium, Schinus e Tapirira) (figuras 6-7), Asteraceae (figura 8), Citrus (Rutaceae), Cocos (Arecaeae) e Poaceae (figura 9) também ocorreram na maioria das amostras, mas sempre com percentuais mais baixos (tabela 1, figura 14). Também foram observados tricomas, epiderme vegetal e hifas de fungos nas amostras analisadas (figuras 10-13).

O tipo polínico Eucalyptus esteve presente como pólen dominante (> 45\%) em nove amostras, das quais três eram de Guaratiba, uma de Campo Grande e cinco de Sepetiba. Barth (1998) e Barth et al. (1999) também observaram a presença de Eucalyptus como pólen dominante em duas outras amostras de própolis do Estado do Rio de Janeiro, bem como Barth \& Luz (2003) para amostras de geoprópolis deste Estado. Os dados indicam que os locais de coleta das amostras de própolis apresentavam arborização com essas espécies exóticas.

O cultivo de Eucalyptus é muito comum em diversas áreas do Rio de Janeiro, e suas espécies são muito utilizadas pelas abelhas Apis mellifera na busca por recursos alimentares como comprovado por Luz et al. (2007b) ao analisar amostras de mel e bolotas de pólen da região serrana do Estado onde este tipo polínico também foi dominante. Em dez localidades do Chile, Montenegro et al. (2001) concluíram que Eucalyptus globulus foi 
용

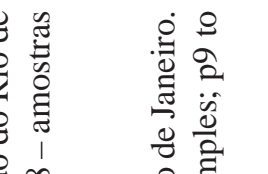

䨔品 串空

되 तै

चृ

要

苂

要

준

Iี

.

告运

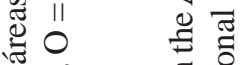

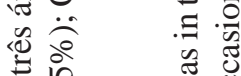

ㄴำ

ज

过苞

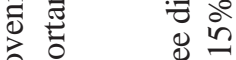

일 至

守守

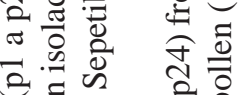

ق

气 득

응

前草

ช ㅇำ

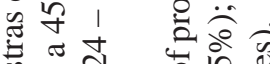

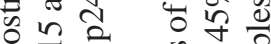

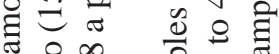

웅

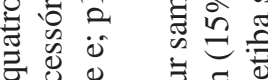

ש

䓫

苛总

व $\varangle$ चี

ஸ $\ddot{2} \cup$

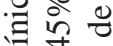

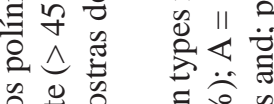

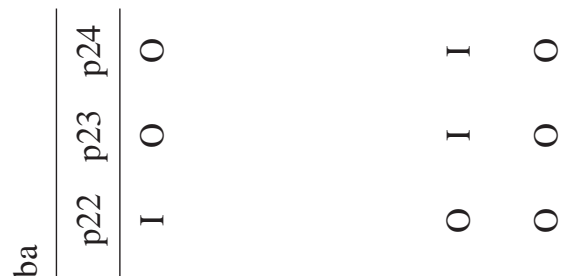

节

ลี 00

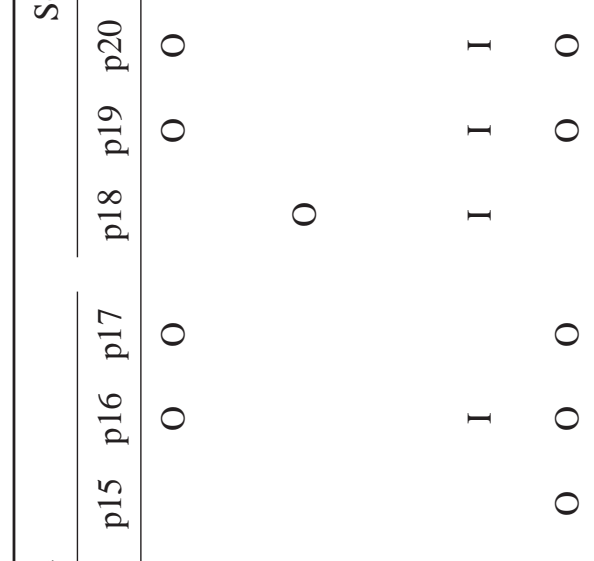

艺

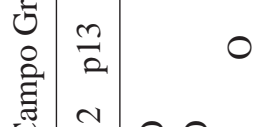

0

00

$\exists 0$

음 0

g. 0

-

$\bigcirc$

龸 䒕

冓

등 음

可

일

잉

䟚

요유

뜽 웡

बै월

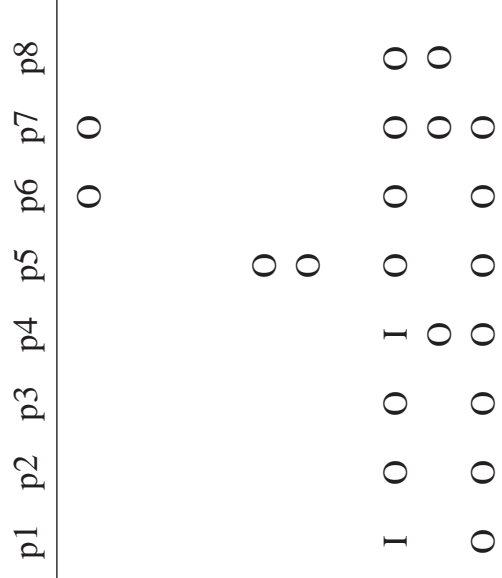

승 空

巳ै छี

苍远

总节。 


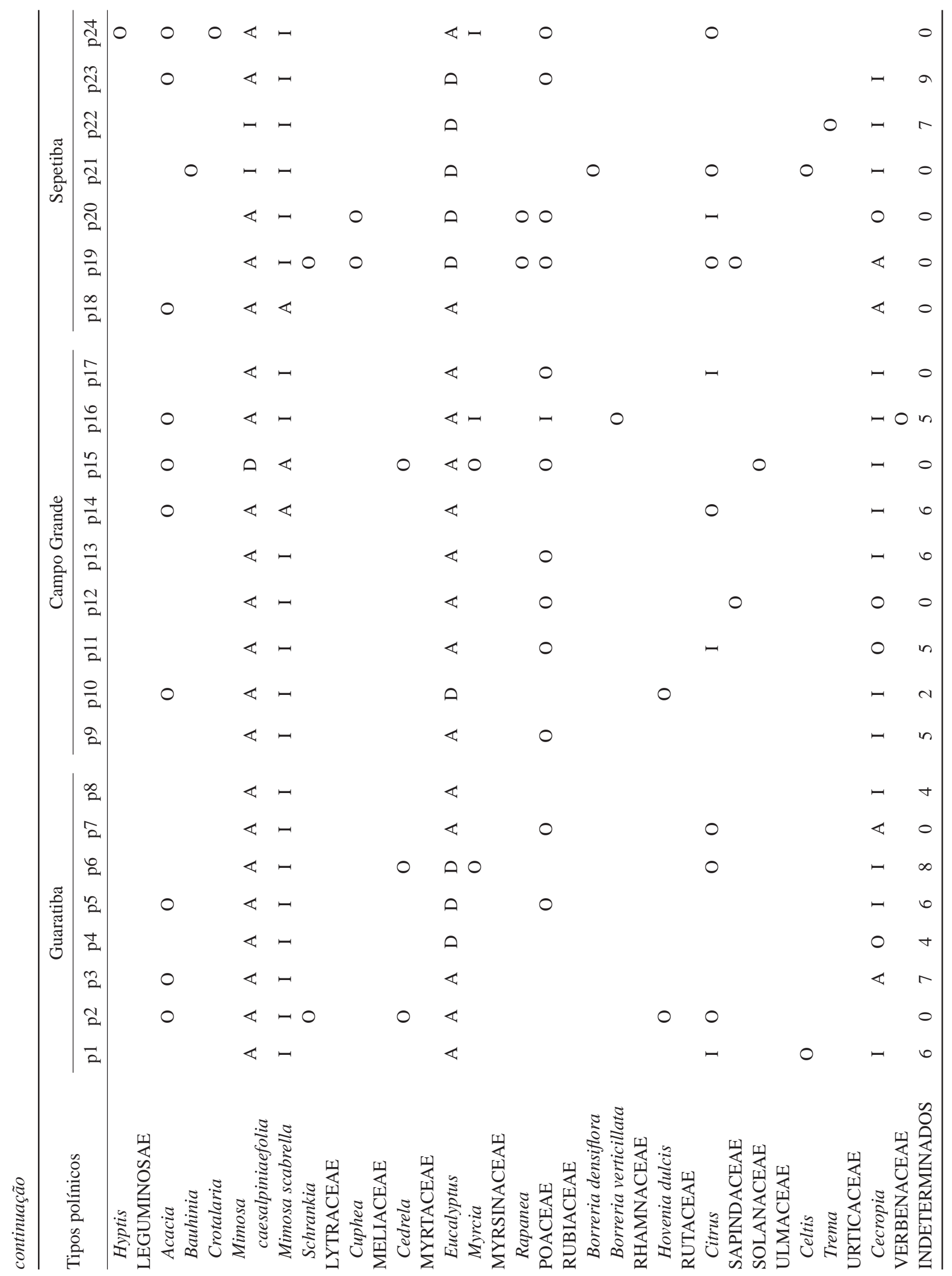



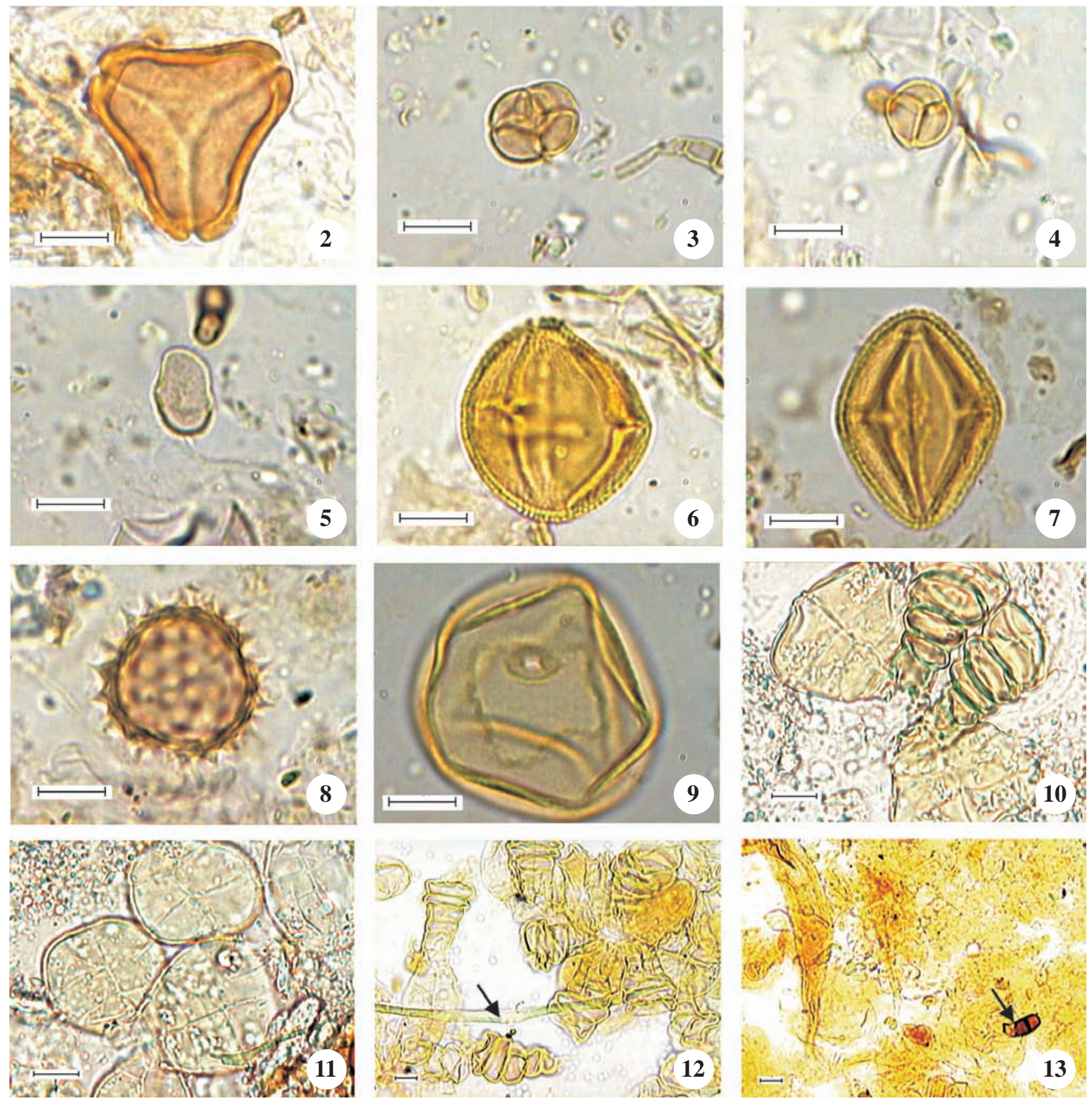

Figuras 2-13. Fotomicrografias dos principais tipos polínicos e tricomas das amostras de própolis marrom da vertente atlântica do Estado do Rio de Janeiro, Brasil. 2. Myrtaceae, Eucalyptus, vista polar. 3-4. Leguminosae. 3. Mimosa caesalpiniaefolia, ditétrade. 4. Mimosa scabrella/Mimosa pudica, tétrade. 5. Urticaceae, Cecropia, vista equatorial. 6-7. Anacardiaceae. 6. Schinus, vista equatorial. 7. Tapirira, vista equatorial. 8. Asteraceae, vista polar. 9. Poaceae, detalhe do poro. 10-11. Tricomas multicelulares. 12. Aspecto geral antes da acetólise evidenciando vários tricomas multicelulares e um único tricoma unicelular alongado (seta). 13. Aspecto geral depois da acetólise evidenciando sedimento orgânico e esporo de fungo (seta). Barra $=10 \mu \mathrm{m}$.

Figures 2-13. Photomicrographs of the main pollen types and trichomes of the brownish propolis from the Atlantic coastal areas in the state of Rio de Janeiro, Brazil. 2. Myrtaceae, Eucalyptus, polar view. 3-4. Leguminosae. 3. Mimosa caesalpiniaefolia, di-tetrad. 4. Mimosa scabrella/Mimosa pudica, tetrad. 5. Urticaceae, Cecropia, equatorial view. 6-7. Anacardiaceae. 6. Schinus, equatorial view. 7. Tapirira, equatorial view. 8. Asteraceae, polar view. 9. Poaceae, detail of pori. 10-11. Multicellular trichomes. 12. General view before acetolysis showing several multicellular trichomes and one long single unicellular trichome (arrow). 13. General view after acetolysis showing organic sediment and spore of fungi (arrow). Bar $=10 \mu \mathrm{m}$. 
uma das fontes de própolis preferida pelas abelhas, de acordo com a presença significativa e constante de seu pólen nas amostras. Através de evidências químicas, a espécie Eucalyptus citriodora foi indicada como uma das possíveis fontes de própolis no Brasil (Lopes et al. 2003). Bastos (2001) fez a mesma sugestão pela presença significativa do pólen de Eucalyptus na própolis de Minas Gerais.

O tipo polínico Mimosa caesalpiniaefolia, tendo como representante mais significativo no Estado o “sabiá”, esteve presente como pólen dominante em somente uma das amostras de Campo Grande. No entanto, ocorreu na maioria das amostras como pólen acessório (15 a 45\%). Barth (1998) e Barth et al. (1999) destacaram este tipo polínico como dominante em outras amostras de própolis analisadas do Estado do Rio de Janeiro. Barth et al. (1999) e Barth \& Luz (2003) citam que nas amostras do Rio de Janeiro este tipo polínico é muitas vezes originário de arbustos lenhosos que ocorrem preferencialmente em locais abertos, alagados ou úmidos, sendo uma importante fonte de recursos poliníferos para as abelhas.

O tipo polínico Mimosa scabrella, representado no Estado principalmente pelo "maricá", ocorreu na maioria das amostras como pólen isolado importante (3 a 15\%) e como pólen acessório em duas de Campo Grande e uma de Sepetiba, sendo considerado um importante indicador da vegetação ruderal. A presença deste tipo polínico em sedimentos de amostras de própolis foi também observada por D'Albore (1979) ao analisar amostras provenientes do Brasil.

Luz et al. (2007b) destacaram que os tipos polínicos Cecropia e Cocos são característicos do Estado do Rio de Janeiro sendo seu pólen encontrado principalmente nas amostras de Guaratiba e Sepetiba. Cecropia ocorre em vegetação degradada da mata atlântica e sobre encostas, Cocos ocorre principalmente em áreas litorâneas como cultivo e foi mais frequente nas amostras de Sepetiba.

Espécies da família Asteraceae ocorrem em várias formações vegetais pioneiras do Estado do Rio de Janeiro e seu pólen foi reconhecido em 19 amostras de própolis das três áreas em estudo. São muito procuradas pelas abelhas Apis mellifera para fornecimento de pólen e néctar. Baccharis dracunculifolia DC. (Bastos et al. 2000, Bastos 2001) é procurada pelas abelhas para coleta de resina no forrageamento da própolis verde.

O pólen de Poaceae estava presente em 13 das amostras de própolis analisadas, indicando também a presença de áreas degradadas e com vegetação pioneira (Menezes \& Araújo 2004, 2005, Menezes et al. 2005).
A presença do pólen de Citrus em 11 das 24 amostras de própolis analisadas indicou que, embora em baixa escala, ocorria o cultivo de frutas cítricas na região.

Os tipos polínicos da família Anacardiaceae estiveram presentes em 16 amostras sendo que Schinus terebinthifolius Raddi possui ampla distribuição em restingas do litoral brasileiro (Afonso et al. 2007), enquanto é comum encontrar Tapirira guianensis Aubl. e Astronium fraxinifolium Schott ex Spreng. nas formações florestais da mata atlântica (Peixoto et al. 2004, Menezes \& Araújo 2004, 2005). Análises do pólen e outras estruturas vegetais da própolis do Cerrado brasileiro sugerem que as abelhas coletam resina de Schinus spp. (Santos et al. 2003) e de Astronium spp. (Bastos 2001).

Nas amostras de própolis de Sepetiba, foram observados assembléias polínicas características da restinga (Schinus), mata atlântica (Bauhinia, Celtis, Rapanea), vegetação espontânea de áreas degradadas (Trema, Acacia, Borreria densiflora, Hyptis e Mimosa scabrella/Mimosa pudica), arbustos de áreas úmidas e alagadas (Mimosa caesalpiniaefolia e Combretum/ Melastomataceae), cultivo (Citrus, Cocos e Eucalyptus) e ampla distribuição (Anacardiaceae, Asteraceae, Cecropia, Crotalaria, Euphorbiaceae, Myrcia, Poaceae e Sapindaceae) (figura 14).

Em Guaratiba ocorreram tipos polínicos representantes de palmeiras do litoral (Astrocaryum), vegetação espontânea de áreas degradadas (Acacia, Gochnatia, Mimosa scabrella/Mimosa pudica), mata atlântica (Casearia, Cedrela, Celtis), arbustos de áreas úmidas e alagadas (Mimosa caesalpiniaefolia), cultivo (Citrus, Cocos, Eucalyptus e Hovenia dulcis) e de ampla distribuição (Anacardiaceae, Apocynaceae, Cecropia, Euphorbiaceae, Myrcia e Poaceae) (figura 14).

Na região de Campo Grande, ocorreram elementos de mata atlântica (Astronium e Mansoa/Sparattosperma), vegetação espontânea de áreas degradadas (Acacia, Eupatorium, Mimosa scabrella/Mimosa pudica), arbustos de áreas úmidas e alagadas (Mimosa caesalpiniaefolia e Combretum/Melastomataceae), cultivo (Citrus, Cocos, Eucalyptus e Hovenia dulcis) e ampla distribuição (Anacardiaceae, Borreria verticillata, Cecropia, Euphorbiaceae, Myrcia, Poaceae, Sapindaceae, Solanaceae, Tabebuia e Verbenaceae) (figura 14).

Foram constantes em todas as três localidades os elementos de vegetação espontânea de áreas degradadas (Mimosa scabrella/Mimosa pudica), arbustos de áreas úmidas e alagadas (Mimosa caesalpiniaefolia) e cultivo (Citrus, Cocos e Eucalyptus) além de elementos que se caracterizam por possuírem ampla distribuição 


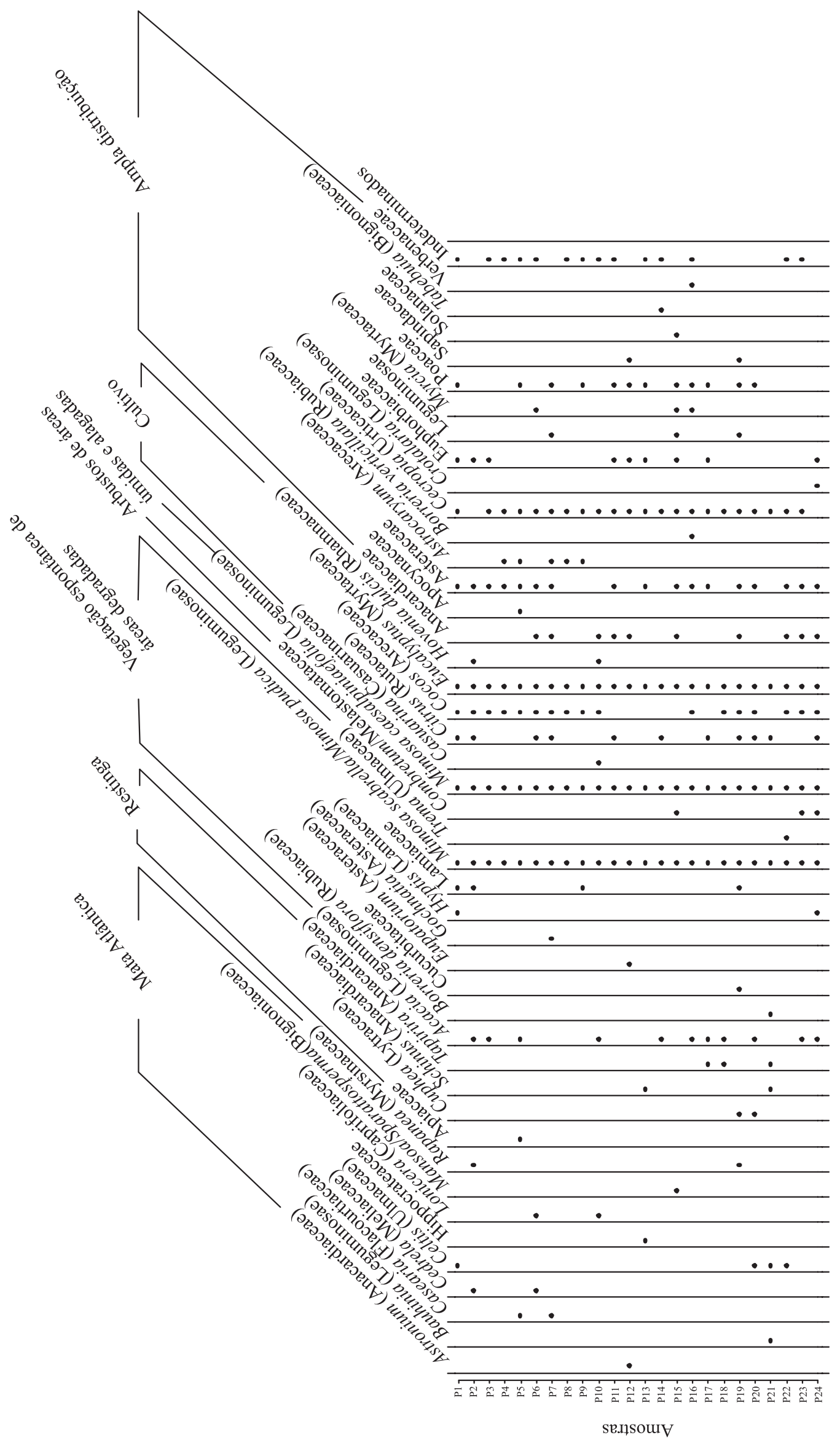


(Anacardiaceae, Cecropia, Euphorbiaceae, Myrcia, Poaceae) (Peixoto et al. 2004, Menezes \& Araújo 2004, 2005, Menezes et al. 2005, Afonso et al. 2007) (figura 14). A análise palinológica da própolis marrom da vertente atlântica do Rio de Janeiro referenciou principalmente a vegetação alterada da grande região de origem.

Os tipos polínicos das amostras de própolis do Rio de Janeiro foram ordenados pela ACP (Análise dos Componentes Principais) de acordo com a semelhança em suas ocorrências, reunindo nos dois primeiros eixos $88,76 \%$ de variabilidade total dos dados e grande similaridade entre as amostras. O primeiro eixo resumiu 85,46\% da variância em função dos valores altamente relacionados principalmente com relação a Eucalyptus e Mimosa caesalpiniaefolia que foram os principais componentes para a ordenação, tanto por apresentarem elevada contagem quanto por estarem presentes em todas as amostras. Mimosa scabrella, Cecropia e Cocos também influenciaram na ordenação por estarem presentes na maioria das amostras. De modo diferente foi o comportamento dos tipos polínicos ordenados no lado direito do primeiro eixo e que apresentaram os menores valores nas contagens e/ou não participaram em todas as amostras (figura 15).

A percentagem de similaridade mostrada no dendrograma foi muito alta entre as amostras $(97,57 \%)$ (figura 16), identificando-se três grupos principais e quatro amostras separadas do restante (p15, p8, p18 e p7). O primeiro grupo do dendrograma (a), na parte esquerda, foi formado por amostras provenientes de Guaratiba e Campo Grande. O segundo grupo no centro do gráfico (b) foi formado por amostras de Guaratiba e Sepetiba. O terceiro grupo (c) foi composto por amostras das três localidades.

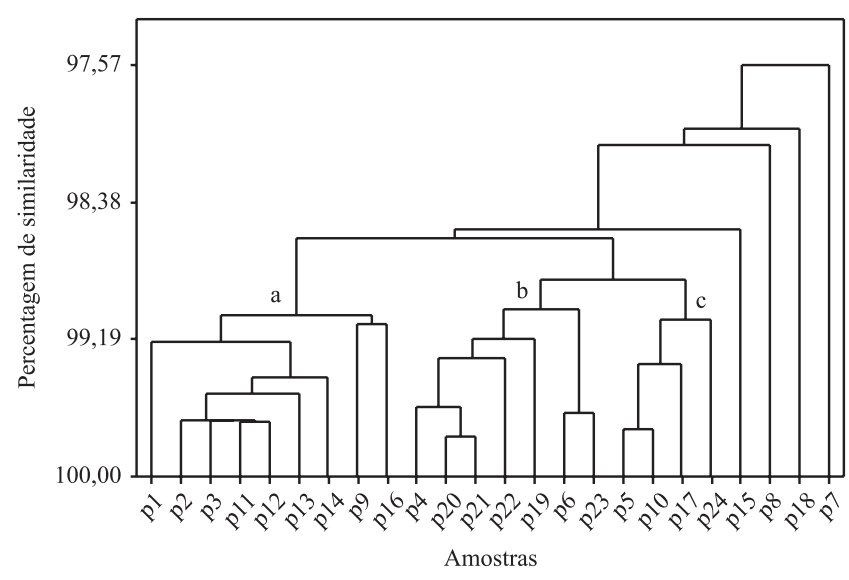

Figura 16. Dendograma de percentagem de similaridade dos grãos de pólen entre as vinte e quatro amostras de própolis marrom da vertente atlântica do Estado do Rio de Janeiro, identificando-se três grupos principais (a, b, c) e quatro amostras separadas do restante (p15, p8, p18 e p7).

Figure 16. Percentage similarity dendrogram carried out on pollen data in the twenty four brownish propolis samples from the Atlantic coastal region in the Rio de Janeiro State, identifying three main groups $(a, b, c)$ and four separate samples (p15, p8, p18 and p7).

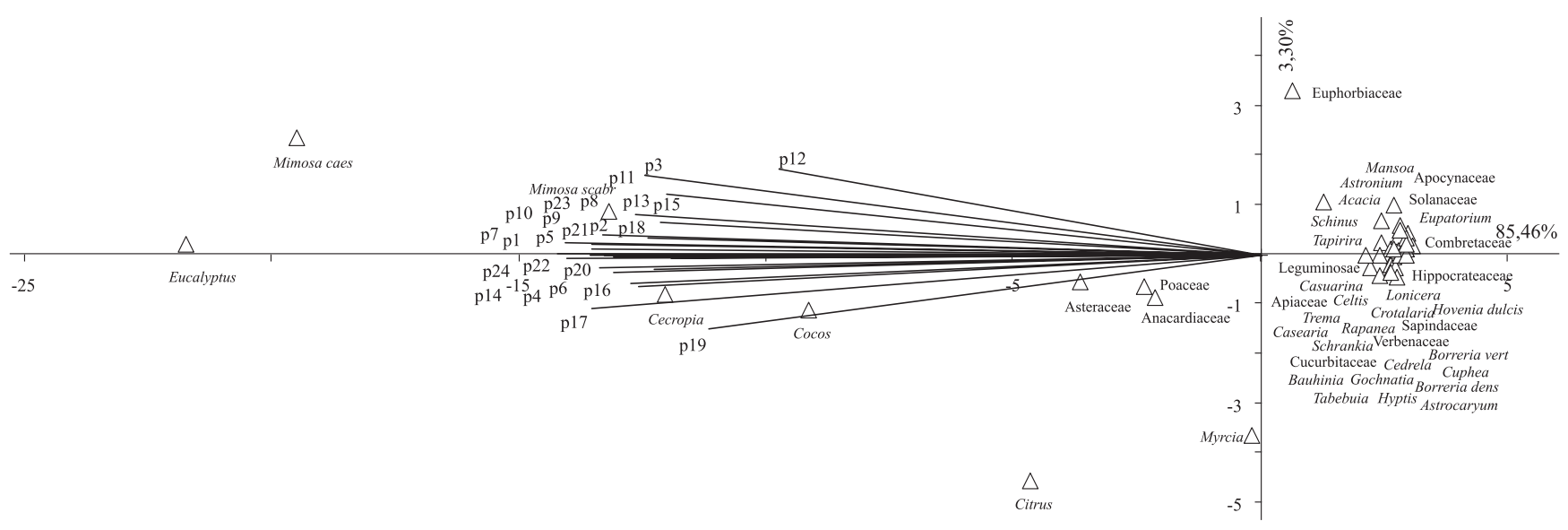

Figura 15. Ordenação pela PCA da composição dos tipos polínicos da própolis marrom da vertente atlântica do Estado do Rio de Janeiro, em função das variáveis numéricas por amostra. Amostras = p1, p2, p3, p4, p5, p6, p7, p8, p9, p10, p11, p12, p13, p14, p15, p16, p17, p18, p19, p20, p21, p22, p23 e p24.

Figure 15. Biplot of PCA for pollen types in the brownish propolis from the Atlantic coastal region in the Rio de Janeiro State, using the absolute value variables per sample. Samples = p1, p2, p3, p4, p5, p6, p7, p8, p9, p10, p11, p12, p13, p14, p15, p16, p17, p18, p19, p20, p21, p22, p23 and p24. 
Assim também ocorreu nas amostras isoladas (duas de Guaratiba, uma de Sepetiba e outra de Campo Grande). Como Guaratiba é área de transição entre Sepetiba (no litoral) e Campo Grande (mais para o interior da baixada), apresenta nos seus limites topográficos tanto a vegetação litorânea quanto a mais interiorizada, gerando grande similaridade das amostras dessa localidade ora com as de Sepetiba, ora com as de Campo Grande.

As assembléias dos tipos polínicos com baixa frequência $(<3 \%$ da soma polínica) apresentaram diferenças entre as amostras das áreas de estudo. Estando presentes, às vezes, em apenas algumas delas, gerou-se 0 isolamento das amostras p7, p8, p15 e p18 no dendograma de similaridade. A amostra p7 foi a única que apresentou Gochnatia, as amostras p8 e p18 apresentaram poucos tipos polínicos e a 15 foi a única que apresentou Mansoa/ Sparattosperma (Bignoniaceae) e Solanaceae.

Os tipos polínicos com baixa frequência nas amostras de própolis podem ser indicativos de espécies botânicas propícias à coleta de resina, como visto em Bastos (2001) e, portanto, à produção de própolis, tais como várias espécies de Anacardiaceae e Asteraceae no presente trabalho. Por isso, quanto mais detalhada for a análise polínica da própolis, incluindo maior número de amostras analisadas por localidade e levando-se também em consideração os tipos polínicos classificados como isolados ocasionais, maior será a resolução fitogeográfica e a contribuição para a interpretação da flora de procedência.

Nas amostras de própolis marrom das três localidades estudadas, os tricomas foram caracterizados por apresentarem células glandulares formadas por pedúnculo espiralado sustentando uma cabeça arredondada pluricelular (figuras 10-12). Foram observados em todas as amostras de Campo Grande e Guaratiba, enquanto nas amostras de Sepetiba nenhum tipo de tricoma foi encontrado. Em algumas amostras ocorreu uma quantidade significativa de material orgânico, tais como epidermes vegetais e hifas de fungos (tabela 2, figura 13).

A ausência ou evidente escassez de estruturas anatômicas vegetais em amostras de própolis é comum e pode ser um indicativo de que está ocorrendo coleta de exsudatos disponibilizados em troncos e ramos ou mesmo em estruturas florais sem que no momento da coleta sejam carreados fragmentos de tecidos vegetais (Teixeira et al. 2003). Por outro lado, a presença de certos elementos figurados na própolis pode ocorrer em dependência de determinadas plantas fornecedoras de resina, como visto em Montenegro et al. (2000, 2001) no Chile. No entanto, estes autores consideraram o
Tabela 2. Procedência das amostras de própolis do Estado do Rio de Janeiro e avaliação do sedimento orgânico. ( $-=$ sem presença de elementos; + = poucos elementos; ++ = frequentes elementos; $+++=$ alta quantidade de elementos).

Table 2. Origin of propolis samples from Rio de Janeiro State and evaluation of the organic sediment. ( $-=$ without elements; + = a small amount of elements; $++=$ numerous elements; +++ = high amount of elements).

\begin{tabular}{|c|c|c|c|}
\hline Procedência & Amostras & Tricomas & $\begin{array}{c}\text { Material orgânico } \\
\text { indeterminado }\end{array}$ \\
\hline Guaratiba & p1 & ++ & - \\
\hline Guaratiba & p2 & ++ & - \\
\hline Guaratiba & p3 & + & + \\
\hline Guaratiba & $\mathrm{p} 4$ & + & - \\
\hline Guaratiba & p5 & ++ & ++ \\
\hline Guaratiba & p6 & ++ & - \\
\hline Guaratiba & p7 & ++ & - \\
\hline Guaratiba & p8 & ++ & ++ \\
\hline Campo Grande & p9 & +++ & - \\
\hline Campo Grande & $\mathrm{p} 10$ & +++ & +++ \\
\hline Campo Grande & p11 & ++ & - \\
\hline Campo Grande & p12 & ++ & - \\
\hline Campo Grande & $\mathrm{p} 13$ & ++ & - \\
\hline Campo Grande & p14 & + & - \\
\hline Campo Grande & p15 & ++ & - \\
\hline Campo Grande & p16 & +++ & - \\
\hline Campo Grande & p17 & + & + \\
\hline Sepetiba & p18 & - & - \\
\hline Sepetiba & p19 & - & + \\
\hline Sepetiba & p20 & - & + \\
\hline Sepetiba & p21 & - & + \\
\hline Sepetiba & p22 & - & ++ \\
\hline Sepetiba & p23 & - & + \\
\hline Sepetiba & p24 & - & + \\
\hline
\end{tabular}

parâmetro como secundário no diagnóstico da origem botânica da própolis, pois, dentre as várias espécies de plantas nativas identificadas nas amostras através de fragmentos de folhas, somente Baccharis linearis (Ruiz \& Pav.) Person apresentou níveis de frequência de grãos de pólen estatisticamente significativos, o que foi suficiente para indicá-la como uma das principais fontes de resina na região estudada.

Para Bastos et al. (2000), a sugestão de Baccharis dracunculifolia DC. como principal fornecedora de resina para a própolis verde de Minas Gerais se deve, principalmente, a maciça presença de fragmentos 
epidérmicos com tricomas e ápices foliares característicos dessa espécie e, não pela significativa participação de seu pólen no espectro polínico (menos de 3\%), levando a concluir que a coleta pelas abelhas foi feita quando a planta não estava em floração e as abelhas fazem uso da resina obtida pela danificação de brotos foliares.

A aparente controvérsia desses resultados demonstra a dificuldade existente na certificação da origem botânica da própolis sem que se correlacione os resultados palinológicos e da identificação anatômica das estruturas vegetais com outras ferramentas analíticas.

Agradecimentos-Ao Conselho Nacional de Desenvolvimento Científico e Tecnológico (CNPq) pela concessão de Bolsas de Produtividade em Pesquisa a segunda e terceira autoras (Processos 301525/2009-9 e 301220/2009-3)

\section{Referências bibliográficas}

AFONSO, A.S., MEDEIROS, A.S., NUNES, C.S., RODRIGUES, G.A., NUNES, R.S., TAVARES, L.F.M. \& CONDE, M.M.S. 2007. Florística da vegetação arbustiva aberta na Restinga da Marambaia, RJ. Revista Brasileira de Biociências 5:450-452.

BANSKOTA, A.H., TEZUKA, Y. \& KADOTA, S. 2001. Recent progress in pharmacological research of propolis. Phytotherapy Research 15:1-11.

BARTH, O.M. 1970a. Análise microscópica de algumas amostras de mel. 1. Pólen dominante. Anais da Academia Brasileira de Ciências 42:351-366.

BARTH, O.M. 1970b. Análise microscópica de algumas amostras de mel. 2. Pólen acessório. Anais da Academia Brasileira de Ciências 42:571-590.

BARTH, O.M. 1970c. Análise microscópica de algumas amostras de mel. 3. Pólen isolado. Anais da Academia Brasileira de Ciências 42:747-772.

BARTH, O.M. 1970d. Análise microscópica de algumas amostras de mel. 4. Espectro polínico de algumas amostras de mel do Estado do Rio de Janeiro. Revista Brasileira de Biologia 30:575-582.

BARTH, O.M. 1989. O pólen no mel brasileiro. Editora Luxor, Rio de Janeiro.

BARTH, O.M. 1998. Pollen analysis of Brazilian propolis. Grana 37:97-101.

BARTH, O.M. 2004. Melissopalynology in Brazil: a review of pollen analysis of honeys, propolis and pollen loads of bees. Scientia Agricola 61:342-350.

BARTH, O.M. 2006. Palynological analysis of geopropolis samples obtained from six species of Meliponinae in the Campus of the Universidade de Ribeirão Preto, USP, Brazil. Apiacta 41:71-85.

BARTH, O.M., DUTRA,V.M.L. \& JUSTO, R.L. 1999. Análise polínica de algumas amostras de própolis do Brasil Meridional. Ciência Rural 29:663-667.
BARTH, O.M. \& LUZ, C.F.P. 2003. Palynological analysis of Brazilian geopropolis sediments. Grana 42:121-127.

BASTOS, E.M.A.F. 2001. Origem botânica e indicadores de qualidade da "própolis verde" produzida no Estado de Minas Gerais, Brasil. Tese de doutorado, Universidade de São Paulo, Ribeirão Preto.

BASTOS, E.M.A.F., OLIVEIRA, V.D.C. \& SOARES, A.E.E. 2000. Microscopic characterization of the green propolis, produced in Minas Gerais state, Brazil. Honeybee Science 21:179-180.

D’ALBORE, G.R. 1979. L’origine géographique de la propolis. Apidologie 10:241-267.

ERDTMAN, G. 1952. Pollen morphology and plant taxonomy - Angiosperms. The Chronica Botanica, Waltham, Massachusetts.

GHISALBERTI, E.L. 1979. Propolis: a review. Bee World 60:59-84.

KUJUMGIEV, A., TSVETKOVA, I., SERKEDJIEVA, Y.U., BANKOVA, V., CHRISTOV, R. \& POPOV, S. 1999. Antibacterial, antifungal and antiviral activity of propolis of different geographic origin. Journal of Ethnopharmacology 64:235-240.

LOPES, F.C., BANKOVA, V. \& SFORCIN, J.M. 2003. Effect of three vegetal sources of propolis on macrophages activation. Phytomedicine 10:343-343.

LOUVEAUX, J., MAURIZIO, A. \& VORWOHL, G. 1978. Methods of melissopalinology. Bee World 59:139-157.

LUZ, C.F.P., BARTH, O.M., CANO, C.B., FELSNER, M.L., CRUZ-BARROS, M.A.V., GUIMARÃES, M.I.T.M. \& CORREA, A.M.S. 2007a. Origem botânica do mel e derivados apícolas e o controle de qualidade. In A Botânica no Brasil: pesquisa, ensino e políticas ambientais. (L.M. Barbosa \& N.A. Santos Junior, orgs.). Sociedade Botânica do Brasil, São Paulo, p.592-596.

LUZ, C.F.P., THOMÉ, M.L. \& BARTH, O.M. 2007b. Recursos tróficos de Apis mellifera (Hymenoptera, Apidae) na região de Morro Azul do Tinguá, Estado do Rio de Janeiro. Revista Brasileira de Botânica 1:27-37.

MANRIQUE, A.J. \& SOARES, A.E.E. 2002. Início de um programa de seleção de abelhas africanizadas para a melhoria na produção de própolis e seu efeito na produção de mel. Interciencia 27:312-316.

MARCUCCI, M.C. 1995. Propolis: chemical composition, biological properties and therapeutic activity. Apidologie 26:83-99.

MARCUCCI, M.C., FERRERES, F., GARCIA-VIGUERA, C., BANKOVA, V., DE CASTRO, S.L., DANTAS, A.P., VALENTE, P.H. \& PAULINO, N. 2001. Phenolics compounds from Brazilian propolis with pharmacological activities. Journal of Ethnopharmacoly 74:105-112.

MCCUNE, B. \& MEFFORD, M.J. 1999. PC-ORD. Multivariate analysis of ecological data, version 4.10. MjM Software Design, Oregon. 
MENEZES, L.F.T. \& ARAÚJO, D.S.D. 2004. Regeneração e riqueza da formação arbustiva de Palmae em uma cronosequência pós-fogo na Restinga da Marambaia, Rio de Janeiro, RJ, Brasil. Acta Botanica Brasilica 18:771-780.

MENEZES, L.F.T. \& ARAÚJO, D.S.D. 2005. Formações vegetais da restinga da Marambaia, Rio de Janeiro. In História Natural da Marambaia (L.F.T. Menezes, A.L. Peixoto \& D.S.D. Araujo, orgs.). Editora da Universidade Federal Rural do Rio de Janeiro, Seropédica.

MENEZES, L.F.T., PEIXOTO, A.L.P. \& ARAUJO, D.S.D. 2005. História Natural da Marambaia. Editora da Universidade Federal Rural do Rio de Janeiro, Seropédica.

MINITAB. 2008. Minitab for Windows [Minitab-Inc, USA] Versão 15. English Ink Copyright [C].

MONTENEGRO, G., TIMMERMANN, B.N. \& PENA, R. 2000. Pollen grains and vegetative structures in propolis as indicators of potential drugs in Chilean plants. Phyton - International Journal of Experimental Botany 66: 15-23.

MONTENEGRO, G., PENA, R. \& TIMMERMANN, B.N. 2001. Botanical resources for propolis in an apiary network in Central Chile. Phyton - International Journal of Experimental Botany 50:191-201.

NOGUEIRA NETO, P. 1997. Vida e criação das abelhas indígenas sem ferrão. Editora Nogueirapis, São Paulo.

PEIXOTO, G.L., MARTINS, S.V., SILVA, A.F. \& SILVA, E.L. 2004. Composição florística do componente arbóreo de um trecho de Floresta Atlântica na Área de Proteção Ambiental da Serra da Capoeira Grande, Rio de Janeiro, RJ, Brasil. Acta Botanica Brasilica 18:151-160.
ROUBIK, D.W. \& MORENO, J.E. 1991. Pollen and spores of Barro Colorado Island. Missouri Botanical Garden, St. Louis.

SALATINO, A., TEIXEIRA, E.W., NEGRI, G. \& MESSAGE, D. 2005. Origin and chemical variation of brazilian propolis. eCAM 2:33-38. http://ecam.oxfordjournals. org/cgi/reprint/neh060v1.pdf (acesso em 05/10/2008).

SANTOS, F.A., BASTOS, E.M.A.F., MAIA, A.B.R.A., UZEDA, M., CARVALHO, M.A.R., FARIAS, L.M. \& MOREIRA, E.S.A. 2003. Brazilian propolis: physicochemical properties, plant origin and antibacterial activity on periodontopathogens. Phytotherapy Research 17:285-289.

SHEPHERD, G.J. 1996. Fitopac 1: manual do usuário. Departamento de Botânica, Universidade Estadual de Campinas, Campinas.

TEIXEIRA, E.W., MESSSAGE, D., MEIRA, R.M.S.A. \& SALATINO, A. 2003. Indicadores da origem botânica da própolis, importância e perspectivas. Revisão bibliográfica. Boletim da Indústria Animal 60:83-106.

TREVISOL, R.G., NEVES, L.G., SILVA, R.T. \& VALCARCEL, R. 2002. Análise da colonização vegetal espontânea em ambientes modificados por medidas físicas na recuperação de áreas degradadas. In Anais do V Simpósio Nacional de Recuperação de Áreas Degradadas (A.C. Davide, S.A. Botelho \& S.C.S Rosado, eds.). Sociedade Brasileira de Recuperação de Áreas Degradadas, Belo Horizonte, p.437-439.

WARAKOMSKA, Z. \& MACIEJEWICZ, W. 1992. Microscopic analysis of propolis from Polish regions. Apidologie 23:277-283. 